noseöl, wie es aus den Kochkesseln kommt, ist von roter Farbe und riecht sehr stark nach Faekalien. Der Speck hat während des langen Sommers eine Gährung durchgemacht, wobei Scatol und Indol nebst chromogenen Eiweissstoffen gebildet wurden. Das rohe Oel wird nach dem Kochen in grosse, flache, eiserne Kessel gepumpt und dort dem Regen und der Sonne ausgesetzt. Hier verbleibt es 1--2 Monate und wird so allmählich gebleicht. Zum Schluss ist es ganz hell geworden, aber der Geruch bleibt schlecht und ist sehr schwer $z u$ entfernen. Nach einer ganz neuen Methode ist es gelungen, das Bottlenoseöl ganz geruchlos zu machen (das warme Oel riecht wie Talg). Das gebleichte Bottlenoseöl hat ge- wöhnlich eine Säurezahl von etwa $2,5-3$ und eine Jodzahl von etwa 80 . Auch hier ist $z u$ bemerken, dass das Oel im Allgemeinen viel Sperm enthält, und es ist sehr schwierig, den Sperm so vollständig zu entfernen, dass das Oel bei Abkühlung- keinen Bodensatz zeigt. In Norwegen giebt es -eine oder zwei Fabriken zur Reinigung von Bottlenoseöl, aber die Methoden werden mit solcher Geheimthuerei umgeben, dass nichts Näheres darüber bekannt ist; wahrscheinlich besteht die Reinigung nur in einer Filtration und Klärung des Oeles, jedenfalls wird aber weder die Säurezahl herabgesetzt, noch der Geruch verbessert.

\title{
Ueber das Vorkommen und den Nachweis von Sesamöl im Arachisöle des Handels.
}

Von P. Soltsien, Görlitz.

Tambon hat kürzlich eine Reaction auf Sesamöl bekannt gegeben, ${ }^{1}$ ) welche eine Verbesserung der Baudouin'schen sein sollte; nach einer Mitteilung von $U t z^{2}$ ) liegt die Grenze der Empfindlichkeit dieser Tambonschen Reaction jedoch schon bei einem Gehalte von $2 \%$ Sesamöl. Die Baudouin'sche Reaction ist also empfindlicher. Gelegentlich dieser Veröffentlichung erwähnt Tambon, dass die Fettsäuren von Arachisöl gleichfalls die Baudouin'sche Reaction geben sollen. Diese Angabe war mir sehr überraschend, da mir eine ähnliche noch niemals in der Litteratur vorgekommen war. Es ist zwar bekannt, dass mit tunesischen und einigen anderen Olivenölen, welche man für ganz rein halten musste, schon Baudouin'sche Reactionen erhalten wurden, anderseits aber auch von Milliau darauf hingewiesen, dass gerade die abgeschiedenen Fettsäuren dieser Olivenöle die schwachen Färbungen mit den Baudouinschen Reagens nicht mehr zeigen, dass also auf diese Weise ermittelt werden könne, ob wirklich Verunreinigung mit Sesamöl vorliege oder nicht. Um so sonderbarer muss es erscheinen, dass nach Tambon selbst die Fettsäuren des Arachisöles noch Baudouin'sche Reactionen geben sollen. Ich hegte die Vermutung, dass diejenigen Arachisöle, bei welchen Tambon diese Beobachtung gemacht habe, mit Sesamöl verunreinigt waren, und dass die Tambon'sche Reaction, mit welchen

1) Apotheker-Ztg. 1901, Nr. 16. Rep. d. Chem.Ztg. $190 \overline{1}$, S. 40.

2) Chem. Ztg. 1901, Nr. 38 . die Uebelstände der Baudouin'schen vermieden werden sollen, nur deshalb nicht das gleiche Resultat ergab, weil sie eben weniger empfindlich ist. Untersuchungen von Arachisölen des Handels, welche ich nach dieser Richtung hin vornahm, ergaben, dass dieses Oel in der Regel mit Sesamöl versetzt oder verunreinigt ist. Ich untersuchte 13 Arachisöle verschiedener Provenienz und keines derselben war frei davon; ausserdem wurden mir in 2 Fällen, einmal von einer Firma in Hamburg, welche speciell Arachisöl vertreten soll, das andere $\mathrm{Mal}$ von einer bekannten Berliner Drogen-Grossinandlung die gewünschten garantiert reinen Muster von Arachisöl, welche ich zur Untersuchung als Vergleichsobjekte erbat, nicht geliefert mit dem Bemerken, dass die betr. Firmen das nicht vermöchten.

Die Reactionen auf Sesamöl wurden sowohl mit Furfurolsalzsäure wie mit Zinnchlorür ausgeführt; mehrfach war auch das Verhalten dieser Oele gegen Salzsäure (vom spec. Gew. 1,19) allein controlliert worden, da z. B. bei Olivenölen täuschende Färbungen mit Salzsäure allein beobachtet sein sollen; die Untersuchung der Arachisölproben nach dieser Richtung hin ergab jedoch keine derartigen Färbungen.

Der erste Versuch wurde mit den Fettsäuren eines eigenen Arachisöles angestellt, welches ich bis dahin für rein gehalten hatte. Diese sowohl wie das Oel selbst geben Reactionen, als wenn mindestens $10 \%$ Sesamöl vorhanden wären. Die nächsten 6 Proben waren aus Hamburg bezogen; die ersten beiden 
waren als ff, Arachis-Tafelöle bezeichnet und sollten als Vergleichsobjecte dienen. Sie gaben Reactionen, wie man sie etwa bei Anwesenheit von $15 \%$ Sesamöl erhält. Auf meine ausdrückliche Reklamation hin erhielt ich Oele, welche noch Reactionen gaben wie bei Gegenwart von etwa 0,25 bis $5 \%$ Sesamöl. Es wurde mir mitgeteilt, dass zu den feinsten Arachisölen vielfach in den Fabriken ein $\mathrm{Zu}-$ satz von gleichwertigem Sesamöle gemacht würde und umgekehrt, „u. a., um die Kältebeständigkeit und Bindefähigkeit des Oeles zu Mayonnaisen günstig zu beeinflussen;" bei geringen Arachisölen werde dieser Zusatz nicht gemacht. Die letztere Angabe fand ich nicht bestätigt, denn auch geringwertige, dunkle Oele warmer Pressung erwiesen sich als sesamölhaltig. Die nur geringen Mengen, welche sich teilweise fanden und nur als Verunreinigungen zu betrachten sind, sollen dadurch bedingt sein, dass vielfach Arachisöl nach Sesamöl gepresst wird. - Fernere 2 Proben waren von einer anderen Hamburger Firma als rein bezogen, kalt wie warm gepresst; sie enthielten etwa $3 \%$ Sesamöl, eine Dresdener Probe etwa $2 \%, 2$ Proben aus Hannover, kalt und warm gepresst, etwa $5 \%, 1$ Probe aus Baden 1\%.

Unter diesen Umständen sah ich mich genötigt, behufs Nachprüfung der Tambonschen Angaben selbst Arachisöle darzustellen. Zur Anwendung gelangten etwa 15 Jahre alte Rufisque- und Kamerun-Nüsse und frischere Rufisque-Nüsse. Die Samen wurden mit Petroläther extrahiert. Die auf diese Weise gewonnenen Oele gaben $\mathrm{k}$ e in e Furfurolsalzsäurereaction. Es treten allmählich nur schwache bräunlich-gelbe Färbungen auf, die kaum einen Stich in's Rötliche zeigten und mit Sesamölreactionen nichts gemein hatten. Schon $0,25 \%$ Sesamöl geben eine ganz andere Reaction. - Was nun die Prüfung der aus diesen Oelen abgeschiedenen Fettsäuren auf Sesamöl anlangt, so ist eigentlich selbstverständlich, dass wenn die Oele selbst keine Reaction geben, die Fettsäuren sie auch nicht geben werden. Weshalb Tambon nur die Reactionen der Fettsäuren des Arachisöles, nicht diejenigen des Oeles selbst erwähnt, ist unverständlich, wenn er nicht annimmt, dass sich die Arachisölfettsäuren von denjenigen anderer in Frage kommender Oele unterscheiden. Aus dem Oele der frischeren Rufisque-Nüsse wurden jedoch, der Vollständigkeit des Versuches wegen, auch die Fettsäuren abgeschieden und mit Furfurolsalzsäure geprüft: der Erfolg war, wie zu erwarten, ein vollständig negativer.

Hierbei möchte ich bemerken, dass die bekannte Milliau'sche Controlle der Baudouin'schen Reaction mir auf Grund folgender Betrachtung nicht stichhaltig erscheint. Enthält ein Arachis- oder Olivenöl thatsächlich S puren von Sesamöl, so dass man nur schwache Baudouin'sche Reactionen damit erhält, so können diese in den Fettsäuren naturgemäss bisweilen nicht mehr nachweisbar sein und zwar dann nicht mehr, wenn bei Abscheidung der Fettsäuren aus den Seifen, wie das wohl gewöhnlich unwillkürlich geschehen wird, die verwendete Säure überschüssig angewendet wird, da der die Sesamölreaction bedingende Stoff gerade in verdünnten Mineralsäuren, auch Essigsäure und anderen, löslich ist und durch Ausschüttelungen damit den fetten Oelen besonders dann leicht entzogen werden kann, wenn er nur in Spuren vorhanden ist; im vorliegenden Falle würden diese Spuren wohl in dem sauren Wasser bleiben und nicht in die Fettsäuren übergehen. Sind mehr als Spuren von Sesamöl vorhanden, so geben die Fettsäuren allerdings noch eine Reaction; $1 \%$ Sesamöl ist, nach Milli au verfahren, schon sehr deutlich nachweisbar.

Zinnchlorür ist zur Prüfung der Fettsäuren auf Sesamöl leider nicht zu verwenden, da dasselbe auf die Fettsäuren sehr heftig unter Bräunung einwirkt. Schon bei Anwesenheit von viel freien Fettsäuren, z. B. in alten Oelen, machen sich mit diesem Reagens störende Braunfärbungen bemerkbar, ja es können sogar Rosafärbungen damit erhalten werden. Ich habe eine solche selbst bei dem aus den frischeren Rufisque-Nüssen hergestellten Oele bemerkt. Diese Färbungen unterscheiden sich zwar von den Sesamölreactionen dadurch, dass sie viel langsamer eintreten, immerhin wendet man in solchen Fällen besser Furforolsalzsäure an oder controlliert eine schwache mit Zinnchlorür erhaltene Färbung mit diesem Reagens, wie man in anderen Fällen eine Furfurolsalzsäurereaction mit Zinnchlorür controllieren muss. 